\title{
Completeness in affine and statistical geometry
}

\author{
Barbara Opozda ${ }^{1}$
}

Received: 8 April 2020 / Accepted: 6 January 2021 / Published online: 10 February 2021

(C) The Author(s) 2021

\begin{abstract}
We begin the study of completeness of affine connections, especially those on statistical manifolds or on affine hypersurfaces. We collect basic facts, prove new theorems and provide examples with remarkable properties.
\end{abstract}

Keywords Affine connection · Geodesic $\cdot$ Completeness $\cdot$ Affine hypersurface $\cdot$ Statistical structure

Mathematics Subject Classification Primary: 53C05 $\cdot 53 \mathrm{C} 22 \cdot 53 \mathrm{~A} 15$

\section{Introduction}

Although affine hypersurfaces have been studied for more than 100 years, the completeness of affine connections naturally appearing on such hypersurfaces was considered, to the knowledge of the author, only in [7] and [8]. In the literature of affine differential geometry, the affine completeness is always meant as the completeness of the affine metric. There are a few very famous theorems in this respect in the case of Blaschke hypersurfaces, like theorems of Blaschke, Calabi, Cheng-Yau, TrudingerWang, see, e.g., [2, 3, 12]. An extensive overview of results from this area can be found in [6]. It is worth to note that the most beautiful results dealing with completeness of the Blaschke metric were proved (sometimes after many attempts) many years after inventing affine differential geometry. As regards the completeness of the induced affine connection (which is usually non-metrizable), it was noticed by Nomizu that the two notions of completeness, that is, the completeness of the affine metric and the one of the induced connection, are independent in general. Namely, in [7] he gave an example of a Blaschke surface in $\mathbf{R}^{3}$ whose Blaschke metric is incomplete, but the induced connection (actually metrizable in this case) is complete. In [8], there is another example (taken from [11]) of a Blaschke surface whose Blaschke metric and the induced connection are both incomplete. These examples are everything which have been known (by now and according to the author's knowledge) about completeness of the induced affine connections. In particular, problems similar to those answered by the

Barbara Opozda

barbara.opozda@im.uj.edu.pl

1 Faculty of Mathematics and Computer Science UJ, ul. Łojasiewicza 6, 30-348 Cracow, Poland 
famous theorems mentioned above and dealing with the induced connections have not been touched yet.

The aim of this paper is to initiate the study of completeness of affine connections appearing in the theory of hypersurfaces as well as in more general situations.

First of all, we prove some positive and negative results on completeness of the induced connections on hypersurfaces. In particular, we prove that on a centroaffine ovaloid the induced connection is complete. Of course, affine connections on compact manifolds do not have to be complete. We also observe that on an affine hypersurface with parallel cubic form the induced connection is not complete unless the induced structure is trivial. In fact, we prove this result in a more general setting, namely for statistical structures on abstract manifolds and with the assumption weaker than that about parallel cubic form. The class of hypersurfaces with parallel cubic form is rich of examples and important in affine differential geometry. Blaschke hypersurfaces satisfying this condition were classified in [4].

Very little is also known about complete statistical connections. The study of complete statistical connections on abstract manifolds was initiated by Noguchi in [9]. He gave a procedure of producing complete statistical connections on complete Riemannian manifolds, see Theorem 4.5. In this paper, we prove new results on completeness of statistical connections. For instance, we prove that on a topological sphere a Riccinon-degenerate projectively flat statistical connection is complete.

We also provide some facts dealing with completeness of affine connections in general. It turns out that using a cubic form defined by a given affine connection and some additionally chosen metric (not necessarily related to the connection) is helpful just as in the theory of affine hypersurfaces or in the geometry of statistical structures.

We also provide examples illustrating the theorems and importance of the imposed assumptions.

The theory of statistical structures is an essential generalization of the theory of equiaffine hypersurfaces. The class of affine hypersurfaces is a small subclass in the category of statistical manifolds. But it is as important for the geometry of statistical manifolds as the theory of Riemannian hypersurfaces of space-forms for the geometry of Riemannian manifolds. In particular, the geometry of affine hypersurfaces is much more developed than the geometry of statistical manifolds. It provides a lot of examples and allows to use the reach technique of the induced objects which makes the considerations more imaginable. For example, Hessian manifolds, which are very important in the theory of statistical structures, are (from the local viewpoint) nothing but improper equiaffine spheres. All sorts of affine spheres have been intensively studied in affine differential geometry, because they are numerous and, in general, non-trivial.

Statistical structures have been know in geometry as Codazzi pairs for more than a hundred years. It is a nice phenomenon that the structures appear in various topics, in which they are often explored anew. This is the case of statistical structures. The theory of statistical structures invented by statisticians in the 80-ties of the XX-th century soon started to need geometry. The geometry of Codazzi pairs was needed. It happened that well-known facts were rediscovered. Therefore, geometers joined the topic and started a new line of research, independent of possible applications in statistics. The idea is to built a geometrical theory, which might be useful for statisticians on one hand and, on the other hand, will be interesting from the viewpoint of pure geometry. The present paper intends to follow this idea. 


\section{Preliminaries}

In affine differential geometry and statistical geometry, there is a big ambiguity in terminology. Therefore, in this section we briefly introduce all notions appearing in this paper, provide basic information on them and fix terminology. All details for this section can be found, for instance, in $[6,8,10]$ and [1].

By a statistical structure on a manifold $M$, we mean a pair $(g, \nabla)$, where $g$ is a positive definite metric tensor field and $\nabla$ is a torsion-free connection (called a statistical connection) on $M$ such that the cubic form $\nabla g$ is symmetric for all arguments. Such a pair used to be also called a Codazzi pair. Denote by $\hat{\nabla}$ the Levi-Civita connection for $g$ and by $K$ the difference tensor between $\nabla$ and $\hat{\nabla}$, that is,

$$
K_{X} Y=\nabla_{X} Y-\hat{\nabla_{X} Y}
$$

where $X, Y$ are vector fields on $M . K(X, Y)$ will stand for $K_{X} Y$. The (1,2)-tensor field $K$ is symmetric and symmetric relative to $g$. Alternatively, we can use the symmetric cubic form $A$ defined as $A(X, Y, Z)=g(K(X, Y), Z))$. One easily sees that $\nabla g=-2 A$. It is clear that a statistical structure can be defined as a pair $(g, A)$, where $g$ is a Riemannian metric and $A$ is a symmetric cubic form. A statistical structure is called trivial if $\nabla=\hat{\nabla}$, (equiv. $K \equiv 0$ or $A \equiv 0)$.

Of course, one can consider the difference tensor $K=\nabla-\hat{\nabla}$ and the cubic forms $\nabla g$ and $A(X, Y, Z)=g(K(X, Y), Z)$ for any connection $\nabla$. If the connection $\nabla$ is torsionfree, then $K$ is symmetric and not necessarily symmetric relative to $g$. We always have $\nabla g(X, Y, Z)=-A(X, Y, Z)-A(X, Z, Y)$. In particular, $\nabla g(X, X, X)=-2 A(X, X, X)$ for any $X$.

Let $(g, \nabla)$ be a statistical structure. The dual connection $\bar{\nabla}$ for the statistical connection $\nabla$ is defined by formula

$$
X g(Y, Z)=g\left(\nabla_{X} Y, Z\right)+g\left(X, \bar{\nabla}_{X} Z\right) .
$$

The dual connection $\bar{\nabla}$ is torsion-free and the pair $(g, \bar{\nabla})$ is a statistical connection. The difference tensor for the dual connection $\bar{\nabla}$ is equal to $-K$, that is, $\bar{\nabla}=\hat{\nabla}-K$.

Let $R, \bar{R}, \hat{R}$ stand for the curvature tensor for $\nabla, \bar{\nabla}$ and $\hat{\nabla}$ respectively. The corresponding Ricci tensors for $\nabla$ and $\bar{\nabla}$ will be denoted by Ric and $\overline{\text { Ric }}$. These Ricci tensors do not have to be symmetric, but they are simultaneously symmetric. Note that Ric is symmetric if and only if $d \tau=0$, where $\tau(X)=\operatorname{tr} K_{X}$.

The $(0,4)$-tensor field $g(R(X, Y) Z, W)$ is not, in general, skew-symmetric for $Z, W$. The class of statistical structures whose curvature tensor fulfills this symmetry condition is very rich, although the condition is very strong. Such structures appear in many situations. In particular, they exist on affine spheres, which constitute the most distinguished class of affine hypersurfaces. We have

Lemma 2.1 Let $(g, \nabla)$ be a statistical structure. The following conditions are equivalent:

1) $R=\bar{R}$,

2) $\hat{\nabla} K$ is symmetric (equiv. $\hat{\nabla} A$ is symmetric),

3) $g(R(X, Y) Z, W)$ is skew-symmetric relative to $Z, W$. 
A statistical structure satisfying one of the above conditions is called conjugate symmetric. Note that the conjugate symmetry implies the symmetry of Ric. We shall need

Proposition 2.2 Let $(g, \nabla)$ be a conjugate symmetric statistical structure. The connection $\nabla$ is projectively flat if and only if $\bar{\nabla}$ is projectively flat.

Proof Since the statistical structure is conjugate symmetric, the Ricci tensors for $\nabla$ and $\bar{\nabla}$ are identical and symmetric. Consider first the case where $\operatorname{dim} M>2$. A Ricci-symmetric torsion-free connection is projectively flat if and only if its curvature tensor is of the form

$$
R(X, Y) Z=\Phi(Y, Z) X-\Phi(X, Z) Y
$$

for some symmetric $(0,2)$-tensor field $\Phi$. Since $R=\bar{R}$, the connections $\nabla$ and $\bar{\nabla}$ are simultaneously projectively flat. Assume now that $\operatorname{dim} M=2$. In this case, the projective flatness of a connection $\nabla$ is equivalent to the symmetry of the cubic form $\nabla$ Ric. Assume $\nabla$ Ric is symmetric. Since $R$ satisfies 3 ) from Lemma 2.1, on a 2-dimensional manifold we have

$$
R(X, Y) Z=k\{g(Y, Z) X-g(X, Z) Y\},
$$

where $k$ is the sectional $\nabla$-curvature of the statistical structure, see [10]. On the other hand, we always have

$$
R(X, Y) Z=\operatorname{Ric}(Y, Z) X-\operatorname{Ric}(X, Z) Y .
$$

It follows that Ric $=k g$. We now have $\overline{\nabla \text { Ric }}=\bar{\nabla}$ Ric $=\nabla$ Ric $-2 K$ Ric $=\nabla$ Ric $-2 k(K g)$, where $K$ acts on $g$ as a differentiation. Since $K g$ is a symmetric cubic form, we get the desired condition.

We shall now briefly recall the way in which statistical structures appear on locally strongly convex affine hypersurfaces.

Let $f: M \rightarrow \mathbf{R}^{n+1}$ be an immersed hypersurface. For simplicity assume that $M$ is connected and orientable. Let $\xi$ be a transversal vector field for the immersion $f$. We define the induced volume form $v_{\xi}$ on $M$ as follows

$$
\nu_{\xi}\left(X_{1}, \ldots, X_{n}\right)=\operatorname{det}\left(f_{*} X_{1}, \ldots, f_{*} X_{n}, \xi\right) .
$$

We also have the induced connection $\nabla$ (torsion-free) and the second fundamental form $g$ (symmetric) defined by the Gauss formula

$$
D_{X} f_{*} Y=f_{*} \nabla_{X} Y+g(X, Y) \xi
$$

where $D$ is the standard flat connection on $\mathbf{R}^{n+1}$. A hypersurface is called non-degenerate if $g$ is non-degenerate. If the hypersurface is locally strongly convex, $g$ is definite. By multiplying $\xi$ by -1 , if necessary, we can assume that $g$ is positive definite. A transversal vector field is called equiaffine if $\nabla v_{\xi}=0$. This condition is equivalent to the symmetry of the cubic form $\nabla g$. In particular, if the hypersurface is locally strongly convex, $(g, \nabla)$ is a statistical structure. It follows that for a statistical structure obtained on a hypersurface by a choice of a transversal vector field, the Ricci tensor of $\nabla$ is automatically symmetric. A hypersurface equipped with an equiaffine transversal vector field is called an equiaffine hypersurface. If $\xi$ is an equiaffine transversal vector field, the Weingarten formula looks as follows 


$$
D_{X} \xi=-f_{*} \mathcal{S} \mathcal{X}
$$

The $(1,1)$-tensor field $\mathcal{S}$ is called the shape operator for $\xi$. It is symmetric relative to $g$. We have the Gauss equation

$$
R(X, Y) Z=g(Y, Z) \mathcal{S X}-g(X, Z) \mathcal{S Y} .
$$

For an equiaffine hypersurface $f$ we define the conormal map

$$
\bar{f}: M \rightarrow\left(\mathbf{R}^{n+1}\right)^{*} \backslash\{0\}
$$

as follows

$$
\bar{f}(x)\left(\xi_{x}\right)=1, \quad(\bar{f}(x))_{\mid f_{*}\left(T_{x} M\right)} \equiv 0 .
$$

If $f$ is non-degenerate, then $\bar{f}$ is an immersion. In this case, for each $x \in M$ the conormal vector $\bar{f}_{x}=0 \bar{f}_{x}$ is transversal to $\bar{f}$. We equip the immersion $\bar{f}$ with the equiaffine transversal vector field $-\bar{f}$. Again, we receive the induced objects on $M$. In particular, the induced connection turns out to be the dual connection for $\nabla$ relative to $g$. The second fundamental form for the conormal immersion is equal to $g(S \cdot, \cdot)$. The Gauss equation for the conormal immersion is the following

$$
\bar{R}(X, Y) Z=g(Y, \mathcal{S Z}) X-g(X, \mathcal{S Z}) Y .
$$

It means that the dual connection $\bar{\nabla}$ is projectively flat if $n>2$. The dual connection is also projectively flat for $n=2$.

We shall use the following version of the fundamental theorem in affine differential geometry, see, e.g., [1].

Theorem 2.3 Let $(g, \nabla)$ be a statistical structure on a simply connected manifold $M$. If $\nabla$ is Ricci-symmetric and the dual connection $\bar{\nabla}$ is projectively flat, then there is an equiaffine immersion $f: M \rightarrow \mathbf{R}^{n+1}$ such that $g$ is the second fundamental form and $\nabla$ is the induced connection for $f$.

Among equiaffine hypersurfaces, we distinguish equiaffine spheres. A proper equiaffine sphere is an equiaffine hypersurface for which the affine lines determined by the chosen equiaffine transversal vector field intersect at one point, called the center of the sphere. The center does not belong to the hypersurface, and the equiaffine transversal vector field is a nonzero constant multiple of the position vector field relative to the center. The shape operator for a proper equiaffine sphere is a nonzero constant multiple of the identity. A proper equiaffine sphere is also said to be a centroaffine hypersurface or a hypersurface with a centroaffine normalization. As it was noticed above, if a hypersurface is locally strongly convex, the conormal map is naturally equipped with a centroaffine normalization. By an improper equiaffine sphere, we mean a hypersurface equipped with a constant transversal vector field. In this case, the shape operator vanishes. An equiaffine locally strongly convex hypersurface is an equiaffine sphere (proper or improper) if an only if the induced statistical structure is conjugate symmetric.

On a locally strongly convex hypersurface, we also have the volume form $v_{g}$ determined by $g$. In general, this volume form is not covariant constant relative to $\nabla$. A basic theorem of the classical affine differential geometry says that there is a unique equiaffine transversal vector field $\xi$ such that $v_{\xi}=v_{g}$. This unique transversal vector field is called the affine 
normal vector field or the Blaschke affine normal. The second fundamental form for the affine normal is called the Blaschke metric or the affine metric. A hypersurface endowed with the affine Blaschke normal is called a Blaschke hypersurface. An affine Blaschke sphere (or just an affine sphere) is a Blaschke hypersurface which is an equiaffine sphere. The easiest examples of Blaschke affine spheres are ellipsoids, hyperboloids and paraboloids. More precisely, when we endow an ellipsoid with the centroaffine normalization with the center at the center of the ellipsoid and a transversal vector field being the opposite to the position vector relative to the center, we get a proper Blaschke affine sphere whose induced statistical structure is trivial. Hyperboloids can be treated in a similar way. For an elliptic paraboloid, the Blaschke affine normal is parallel to the axis of the paraboloid (so it is an improper affine sphere) and the induced statistical structure is also trivial. A classical theorem says that the only compact Blaschke affine spheres are ellipsoids with their trivial structure.

\section{Geodesics of affine connections}

Before we study geodesics of statistical connections, we shall collect some facts about geodesics of affine connections in general. Some of the facts are likely known, but we prove all of them. Riemannian metrics which we consider in this section are not related to the connections in any way.

By a $\nabla$-geodesic, we mean a geodesic relative to a connection $\nabla$ parametrized by its affine parameter, if not otherwise stated. A geodesic is called complete if its affine parameter runs from $-\infty$ to $\infty$. A pre-geodesic is a curve, parametrized or unparametrized, which can be parametrized or reparametrized in such a way that it becomes a geodesic. Since geodesics are regular curves, we will consider only regular parametrizations of pre-geodesics.

Recall first the following theorem proved, for instance, in [5] in Chapter III, see Theorem 8.7. For a given connection on $M$ let $\left(x^{1}, \ldots, x^{n}\right)$ be a normal coordinate system with origin at $x_{0} \in M$. Let $\mathcal{U}\left(x_{0} ; \rho\right)$ denote the geodesic ball of radius $\rho$, that is, $\mathcal{U}\left(x_{0} ; \rho\right)=\left\{\left(x^{1}, \ldots, x^{n}\right) \in M:\left(x^{1}\right)^{2}+\cdots+\left(x^{n}\right)^{2}<\rho^{2}\right\}$.

Theorem 3.1 Let $M$ be equipped with a linear connection. For each point $x_{0}$ of $M$ and its normal coordinate system $\left(x^{1}, \ldots, x^{n}\right)$ with origin at $x_{0}$ there is a positive number a such that if $0<\rho<a$, then $\mathcal{U}\left(x_{0} ; \rho\right)$ is geodesically convex and each point from $\mathcal{U}\left(x_{0} ; \rho\right)$ has a normal coordinate neighborhood containing $\mathcal{U}\left(x_{0} ; \rho\right)$.

Using this theorem, one can prove

Proposition 3.2 Let $M$ be equipped with a connection. If $\gamma:(a, b) \rightarrow M$ is a geodesic and there is the limit of $\gamma(t)$ in $M$ for $t \rightarrow b$, then $\gamma$ can be extended as a geodesic beyond $b$.

Proof Let $x_{0}=\lim _{t \rightarrow b} \gamma(t)$ and $\mathcal{U}$ be a normal neighborhood of $x_{0}$ as in Theorem 3.1. In this neighborhood there is $\gamma\left(t_{0}\right)$ for some $t_{0} \in(a, b)$. We can assume that $t_{0}=0$. There is a normal neighborhood $\mathcal{U}^{\prime}$ of $p:=\gamma(0)$ containing $\mathcal{U}$. Take the neighborhood $\exp _{p}^{-1}\left(\mathcal{U}^{\prime}\right)$ of 0 in $T_{p} M$. We have $\beta(t):=\exp _{p}^{-1}(\gamma(t))=\dot{\gamma}(0) t$ for $t \in[0, b)$. Since $x_{0} \in \mathcal{U} \subset \mathcal{U}^{\prime}$, the segment $\beta(t)$ of a straight line can be extended beyond $b$. Hence, the geodesic $\gamma$ can be extended beyond $b$. 
By the same arguments as in the last proof, we get

Proposition 3.3 Let $M$ be equipped with a connection. If $\gamma:(a, \infty) \rightarrow M$ is a geodesic, then there is no limit of $\gamma(t)$ in $M$ for $t \rightarrow \infty$.

We can now reformulate Proposition 3.2 as follows

Proposition 3.4 Let $M$ be equipped with a connection. If $\gamma(t)$ for $t \in(a, b)$, where $b \in \mathbf{R}$ or $b=\infty$ is a pre-geodesic and there is $x_{0}=\lim _{t \rightarrow b} \gamma(t)$ in $M$, then the pre-geodesic can be extended as a pre-geodesic beyond $x_{0}$.

Proof Since we can reparametrize the curve to an affine parameterization, we can assume that $\gamma(t)$ is a geodesic. By Proposition 3.3 we have that $b \in \mathbf{R}$. It is now sufficient to Proposition 3.2 .

Let now $(M, g)$ be a Riemannian manifold. The distance between points $x, y \in M$ will be denoted by $d(x, y)$ and the length of a curve $\gamma$ by $d \gamma$.

Proposition 3.5 Let $\gamma:[a, b) \rightarrow M$, where $b \in \mathbf{R}$, be a $\mathcal{C}^{1}$-curve. If the curve is extendable (as a $\mathcal{C}^{1}$-curve) to $[a, b]$, then $\|\dot{\gamma}\|$ is bounded in $[a, b)$. If $(M, g)$ is complete and $\|\dot{\gamma}\|$ is bounded on $[a, b)$, then there is the limit $\lim _{t \rightarrow b} \gamma(t) \in M$.

Proof The first assertion is trivial. Assume now that $(M, g)$ is complete and $\|\dot{\gamma}\| \leq N$ in $[a, b)$. Let $t_{n} \in[a, b)$ be a sequence converging to $b$. We have

$$
\begin{aligned}
d\left(\gamma\left(t_{n}\right), \gamma\left(t_{m}\right)\right) & \leq \text { the length of } \gamma \text { between } \gamma\left(t_{n}\right) \text { and } \gamma\left(t_{m}\right) \\
& =\int_{t_{n}}^{t_{m}}\|\dot{\gamma}(t)\| d t \leq N\left|t_{m}-t_{n}\right| .
\end{aligned}
$$

Hence, the sequence $\gamma\left(t_{n}\right)$ is a Cauchy one.

Proposition 3.6 Let $\gamma(t)$ for $t \in[a, b)$, where $b \in \mathbf{R}$ or $b=\infty$, be a curve in a complete Riemannian manifold $(M, g)$. If $d \gamma<\infty$, then $\lim \gamma(t)$ for $t \rightarrow$ b exists in $M$.

Proof Let $d \gamma<N<\infty$. The closed ball $\mathcal{B}=\{x \in M: d(x, \gamma(a)) \leq N\}$ is compact and $\operatorname{im} \gamma \subset \mathcal{B}$. Take any sequence $t_{n} \rightarrow b$. There is a subsequence $t_{n_{m}}$ such that the sequence of points $\gamma\left(t_{n_{m}}\right)$ has a limit in $\mathcal{B}$. We now claim that $\gamma(t)$ has a limit for $t \rightarrow b$. Suppose that there are two sequences $t_{n} \rightarrow b$ and $s_{n} \rightarrow b$ such that $\gamma\left(t_{n}\right) \rightarrow p$ and $\gamma\left(s_{n}\right) \rightarrow q$ where $p \neq q$. We can assume that $t_{n}$ and $s_{n}$ are increasing and

$$
t_{1}<s_{1}<t_{2}<s_{2}<t_{3} \cdots .
$$

Take two balls $\mathcal{B}_{1}, \mathcal{B}_{2}$ with centers at $p$ and $q$ respectively, such that $d\left(\mathcal{B}_{1}, \mathcal{B}_{2}\right)>\delta>0$. We can assume that $\gamma\left(t_{n}\right) \in \mathcal{B}_{1}$ and $\gamma\left(s_{n}\right) \in \mathcal{B}_{2}$ for all $n$. Then $\left.d \gamma\right|_{t_{1}} ^{s_{1}}>\delta,\left.d \gamma\right|_{t_{2}} ^{s_{2}}>\delta, \ldots$ It follows that $d \gamma=\infty$, which gives a contradiction.

Proposition 3.7 Let $(M, g)$ be a complete Riemannian manifold and $\nabla$ a connection on $M$. If $r(s)$ is an arc-length parametrization of a maximal $\nabla$-geodesic, then the parameter $s$ runs from $-\infty$ to $\infty$. In particular, every maximal $\nabla$-geodesic has infinite length. 
Proof Assume that the whole domain of $r$ is $(a, b)$, where $b \in \mathbf{R}$. By Proposition 3.5, we know that there exists the limit of $r(s)$ for $s \rightarrow b$. By Proposition 3.4, the pre-geodesic can be extended, which is a contradiction with the maximality. Hence $b=\infty$. For the same reasons $a=-\infty$.

Proposition 3.8 Let $\nabla$ be a connection on a Riemannian manifold $(M, g)$. If $r(s)$ is an arclength parametrization of a $\nabla$-geodesic $\gamma(t)$, then

$$
\nabla_{\dot{r}} \dot{r}=A(\dot{r}, \dot{r}, \dot{r}) \dot{r} .
$$

Moreover, if $t=\varphi(s)$ is the change of the parameters, where $\varphi^{\prime}>0$, then

$$
A(\dot{r}, \dot{r}, \dot{r})=-\frac{d}{d s} \ln \|\dot{\gamma} \circ \varphi\| .
$$

Proof We have

$$
0=\frac{d}{d s} g(\dot{r}, \dot{r})=\nabla g(\dot{r}, \dot{r}, \dot{r})+2 g\left(\nabla_{\dot{r}} \dot{r}, \dot{r}\right)
$$

Since $\nabla_{\dot{r}} \dot{r}$ is parallel to $\dot{r}$, we now have $\nabla_{\dot{r}} \dot{r}=-\frac{1}{2} \nabla g(\dot{r}, \dot{r}, \dot{r}) \dot{r}=A(\dot{r}, \dot{r}, \dot{r}) \dot{r}$.

For proving the second assertion observe that since $r(s)=\gamma(\varphi(s))$ and $\dot{r}(s)=\varphi^{\prime}(s)(\dot{\gamma} \circ \varphi)(s)$, we have

$$
\|(\dot{\gamma} \circ \varphi)(s)\|=\frac{1}{\varphi^{\prime}(s)}=g((\dot{\gamma} \circ \varphi)(s), \dot{r}(s)) .
$$

Consequently

$$
\begin{aligned}
& \|\dot{\gamma} \circ \varphi\|^{\prime}=\frac{d}{d s} g(\dot{\gamma} \circ \varphi, \dot{r}) \\
& \quad=\nabla g(\dot{r}, \dot{\gamma} \circ \varphi, \dot{r}))+g\left(\nabla_{\dot{r}}(\dot{\gamma} \circ \varphi), \dot{r}\right)+g\left(\dot{\gamma} \circ \varphi, \nabla_{\dot{r}} \dot{r}\right) \\
& \quad=\frac{1}{\varphi^{\prime}} \nabla g(\dot{r}, \dot{r}, \dot{r})+g\left(\varphi^{\prime} \nabla_{\dot{\gamma} \circ \varphi}(\dot{\gamma} \circ \varphi), \dot{r}\right)+\frac{1}{\varphi^{\prime}} A(\dot{r}, \dot{r}, \dot{r}) \\
& \quad=-2 \frac{1}{\varphi^{\prime}} A(\dot{r}, \dot{r}, \dot{r})+\frac{1}{\varphi^{\prime}} A(\dot{r}, \dot{r}, \dot{r})=-\frac{1}{\varphi^{\prime}} A(\dot{r}, \dot{r}, \dot{r}) .
\end{aligned}
$$

Take $\Lambda=-\ln \|\dot{\gamma} \circ \varphi\|$. Then

$$
\frac{d}{d s} \Lambda=-\frac{\|\dot{\gamma} \circ \varphi\|^{\prime}}{\|\dot{\gamma} \circ \varphi\|}=\frac{\frac{1}{\varphi^{\prime}} A(\dot{r}, \dot{r}, \dot{r})}{\frac{1}{\varphi^{\prime}}} .
$$

Proposition 3.9 Let $r(s), s \in \mathbf{R}$, be any regular parametrization of a maximal geodesic of some affine connection $\nabla$ on a manifold $M$. Let $\nabla_{\dot{r}} \dot{r}=\Lambda^{\prime} \dot{r}$, where $\Lambda$ is a function bounded from below or above on the whole of $\mathbf{R}$. Then, the geodesic is complete.

Proof Let $t=\varphi(s)$ be an affine parameter of our geodesic, say $\gamma(t)$. We can assume that $\varphi^{\prime}>0$ on $\mathbf{R}$. Then $\gamma(\varphi(s))=r(s), \varphi^{\prime}(\dot{\gamma} \circ \varphi)=\dot{r}$ and consequently 


$$
\nabla_{\dot{r}} \dot{r}=\nabla_{\dot{r}}\left(\varphi^{\prime}(\dot{\gamma} \circ \varphi)\right)=\varphi^{\prime \prime}(\dot{\gamma} \circ \varphi)
$$

Hence $\Lambda^{\prime}=\left(\ln \varphi^{\prime}\right)^{\prime}$ and we can assume that $\varphi^{\prime}=e^{\Lambda}$. If $\Lambda$ is bounded from below, then $\varphi^{\prime}$ is greater than some positive number. Therefore $\varphi(s)_{s \rightarrow \infty} \rightarrow \infty$ and $\varphi(s)_{s \rightarrow-\infty} \rightarrow-\infty$. If $\Lambda$ is bounded from above, then we can change the orientation of the parametrization $r(s)$ and in equation $\nabla_{\dot{r}} \dot{r}=\Lambda^{\prime} \dot{r}$ we replace $\Lambda$ by $-\Lambda$, which is now bounded from below.

Proposition 3.10 Let $\nabla$ be a connection on a complete Riemannian manifold. If a maximal $\nabla$-geodesic has scalar speed bounded (from above), then the geodesic is complete.

Proof By Proposition 3.7, we know that the arc-length parameter of the geodesic runs from $-\infty$ to $+\infty$. Use the same notations and agreements as in Proposition 3.8. By (9) $\varphi^{\prime}$ is greater than some positive number for every $s$. It means that $\varphi(s)_{s \rightarrow \infty} \rightarrow \infty$ and $\varphi(s)_{s \rightarrow-\infty} \rightarrow-\infty$.

For a $\nabla$-geodesic $\gamma(t)$ on a Riemannian manifold we introduce two functions $l(t)=\|\dot{\gamma}(t)\|$ and $u(t)=\frac{\dot{\gamma}(t)}{\|\dot{\gamma}(t)\|}$. We have

$$
\begin{aligned}
& \frac{d}{d t} g(\dot{\gamma}(t), \dot{\gamma}(t))=2 g\left(\hat{\nabla}_{\dot{\gamma}(t)} \dot{\gamma}, \dot{\gamma}(t)\right)=-2 g\left(K_{\dot{\gamma}(t)} \dot{\gamma}(t), \dot{\gamma}(t)\right) \\
& =-2 A(\dot{\gamma}(t), \dot{\gamma}(t), \dot{\gamma}(t))=-2 A\left(\frac{\dot{\gamma}(t)}{\|\dot{\gamma}(t)\|}, \frac{\dot{\gamma}(t)}{\|\dot{\gamma}(t)\|}, \frac{\dot{\gamma}(t)}{\|\dot{\gamma}(t)\|}\right)\|\dot{\gamma}(t)\|^{3} .
\end{aligned}
$$

Hence,

$$
\frac{d}{d t}\left(l^{2}(t)\right)=-2 A(u(t), u(t), u(t)) l^{3}(t)
$$

and therefore

$$
\left(\frac{1}{l}\right)^{\prime}=A(u, u, u)
$$

Assume that the geodesic $\gamma$ is defined on the interval $[a, b)$. We can assume that $a=0$ and $l(0)=\|\dot{\gamma}(0)\|=1$. Assume that

$$
-N \leq A(U, U, U) \leq N
$$

for some nonnegative number $N$ and all $U \in \mathcal{U M}$, where $\mathcal{U M}$ is the unit sphere bundle over $M$. It happens, for instance, if $M$ is compact. We have

$$
\frac{1}{l(t)}=1+A(u(\theta), u(\theta), u(\theta)) t
$$

for every $t \in(0, b)$ and some $\theta \in(0, t)$. Since $A(u(\theta), u(\theta), u(\theta)) \geq-N$, we get

$$
l(t) \leq \frac{1}{1-N t},
$$

if $t<\frac{1}{N}$. The function $[0, b) \ni t \rightarrow \frac{1}{1-N t}$ is positive valued and increasing. Using now Proposition 3.5, we get 
Proposition 3.11 Let $\nabla$ be a connection on a Riemannian manifold $M$ whose metric is complete and (14) holds. Let $\gamma:[0, b) \rightarrow M$ be a $\nabla$-geodesic such that $\|\dot{\gamma}(0)\|=1$. Then, the geodesic can be extended at least up to any parameter $c<\frac{1}{N}$.

Proof Indeed, if $c<\frac{1}{N}$, then the function $l$ is bounded on the interval $[0, c)$.

In contrast with Proposition 3.7 in the following proposition, the Riemannian manifold does not have to be complete.

Proposition 3.12 Let $\nabla$ be a connection on a Riemannian manifold and (14) holds. Every complete $\nabla$-geodesic has infinite length.

Proof By (13) we have $\left(\frac{1}{l}\right)^{\prime} \leq N$. By integrating this inequality on an interval $[0, t]$ we get $\frac{1}{l(t)} \leq N t+1$. Consequently, for every $t \in R^{+}$we have $l(t) \geq \frac{1}{N t+1}$. Integrating this inequality on an interval $[0, t]$ we obtain

$$
d \gamma_{[0, t]} \geq \frac{\ln (N t+1)}{N}
$$

It is easy to find an incomplete metric and its statistical connection whose complete geodesic has finite length.

Example 3.13 Let $M$ be the open strip $\mathbf{R} \times(-L, L) \subset \mathbf{R}^{2}$ endowed with the standard metric $g$, where $L=\frac{\sqrt{\pi}}{2}$. Let $G(t)$ be the anti-derivative of the Gaussian function $e^{-t^{2}}$ with $G(0)=0$. Denote by $(U, V)$ the canonical frame on $M$. Each point of $M$ has the coordinates $(x, G(t))$ for some $t \in \mathbf{R}$. Take any symmetric and symmetric relative to $g(1,1)$-tensor field $K$ such that

$$
K_{(x, G(t))}(V, V)=2 t e^{t^{2}} V .
$$

Let $\nabla=\hat{\nabla}+K$. The piece of a straight line $\gamma(t)=(0, G(t))$ for $t \in(-\infty, \infty)$ is a complete $\nabla$ -geodesic. Of course, it has finite length $\sqrt{\pi}$. Note that (14) is not satisfied.

We shall now give an example of a statistical structure on a compact manifold whose statistical connection is not complete. The example is important for the last section of this paper.

Example 3.14 Take $\mathbf{R}^{2}$ with its standard flat Riemannian structure. Let $U, V$ be the canonical frame field on $\mathbf{R}^{2}$. Define the statistical connection $\nabla$ as follows

$$
\nabla_{U} U=U, \nabla_{U} V=-V, \nabla_{V} V=-U .
$$

The statistical structure can be projected on the standard torus $T^{2}=\mathbf{R}^{2} / \mathbf{Z}^{2}$. Observe that $\hat{\nabla} K=0$ (equivalently $\hat{\nabla} A=0$ ); hence, the structure is conjugate symmetric. For later use, observe also that $\nabla$ is projectively flat and its Ricci tensor is non-degenerate negative definite.

A curve $\gamma(t)=(x(t), y(t))$ is a $\nabla$-geodesic if and only if 


$$
x^{\prime \prime}+\left(x^{\prime}\right)^{2}-\left(y^{\prime}\right)^{2}=0, \quad y^{\prime \prime}-2 x^{\prime} y^{\prime}=0 .
$$

Let $y_{0}$ be a fixed real number. Consider the curve

$$
\gamma(t)=\left(\ln (1-t), y_{0}\right)
$$

for $t \in[0,1)$. It is a $\nabla$-geodesic. We have $\gamma(t) \rightarrow\left(-\infty, y_{0}\right),\|\dot{\gamma}(t)\|=\frac{1}{1-t_{2}} \rightarrow+\infty$ for $t \rightarrow 1$. This geodesic cannot be extended beyond 1 , and its image is closed in $T^{2}$. It means, in particular, that closed geodesics of affine connections do not have to be complete.

\section{Completeness of statistical connections}

The following theorem generalizes Example 3.14.

Theorem 4.1 Let $(g, \nabla)$ be a statistical structure for which $\nabla$ is complete and $(\hat{\nabla} A)(U, U, U, U) \leq 0$ for each $U \in \mathcal{U M}$. Then, the statistical structure must be trivial.

Proof We shall first prove

Lemma 4.2 Let $\gamma(t)$ be a $\nabla$-geodesic. Then,

$$
\begin{aligned}
& (A(u, u, u))^{\prime}(t) \\
& =l(t)[(\hat{\nabla} A)(u(t), u(t), u(t), u(t)) \\
& \left.\quad-3 \sum_{i=2}^{n}\left(A\left(u(t), u(t), e_{i}(t)\right)\right)^{2}\right],
\end{aligned}
$$

where $e_{1}(t)=u(t), e_{2}(t), \ldots, e_{n}(t)$ is an orthonormal basis of $T_{\gamma(t)} M$. In particular,

$$
(A(u, u, u))^{\prime}(t) \leq l(t)(\hat{\nabla} A)(u(t), u(t), u(t), u(t)) .
$$

Proof Using (13), we get

$$
\begin{aligned}
& \hat{\nabla}_{\dot{\gamma}(t)} u=\hat{\nabla}_{\dot{\gamma}(t)} \frac{\dot{\gamma}}{l} \\
& =\left(\frac{1}{l}\right)^{\prime}(t) \dot{\gamma}(t)-\frac{1}{l(t)} K(\dot{\gamma}(t), \dot{\gamma}(t)) \\
& =l(t)[A(u(t), u(t), u(t)) u(t)-K(u(t), u(t))] .
\end{aligned}
$$

Since

$$
\begin{array}{r}
K(u(t), u(t))=\sum_{i=1}^{n} g\left(K(u(t), u(t)), e_{i}(t)\right) e_{i}(t) \\
=\sum_{i=1}^{n} A\left(u(t), u(t), e_{i}(t)\right) e_{i}(t)
\end{array}
$$

for any orthonormal frame $e_{1}(t), \ldots, e_{n}(t)$, we now obtain (using also the symmetry of $A$ ) 


$$
\begin{aligned}
& (A(u, u, u))^{\prime}(t)=\dot{\gamma}(t)(A(u, u, u)) \\
& =(\hat{\nabla} A)(\dot{\gamma}(t), u(t), u(t), u(t))+3 A\left(\hat{\nabla}_{\dot{\gamma}(t)} u, u(t), u(t)\right) \\
& =l(t)\left[(\hat{\nabla} A)(u(t), u(t), u(t), u(t))+3(A(u(t), u(t), u(t)))^{2}\right. \\
& \left.\quad-3\left(A\left(u(t), u(t), e_{1}(t)\right)\right)^{2}-3 \sum_{i=2}^{n}\left(A\left(u(t), u(t), e_{i}(t)\right)\right)^{2}\right] .
\end{aligned}
$$

By setting $e_{1}(t)=u(t)$, we complete the proof of the lemma.

Suppose now that the structure $(g, \nabla)$ is not trivial. Let $\gamma:[0, \infty)$ be a $\nabla$-geodesic such that $A(u(0), u(0), u(0))=-N$, where $N$ is a positive number. The function $\frac{1}{l}$ is smooth and positive on $[0, \infty)$. By Taylor's formula and (13), we have

$$
\frac{1}{l(t)}=\frac{1}{l(0)}-N t+\alpha(\theta) t^{2}
$$

for any $t \in[0, \infty)$, where $\theta \in(0, t)$ and $2 \alpha=(A(u, u, u))^{\prime}$. Take $t_{0}>\frac{1}{l(0) N}$. For $t=t_{0}$, the expression on the right hand side of (25) is negative by Lemma 4.2. This gives a contradiction.

Observe that on a compact $M$ if $(\hat{\nabla} A)(U, U, U, U) \leq 0$ for every $U \in \mathcal{U M}$, then $\hat{\nabla} A=0$ on $M$. It follows from Ros' integral formula. Namely, the formula says that for any covariant tensor field $s$ on a compact Riemannian manifold we have $\int_{\mathcal{U} M}(\hat{\nabla} s)(U, \ldots, U) d U=0$. However, the fact that $\hat{\nabla} A=0$ does not trivialize the situation. First of all, even in the theory of affine hypersurfaces one knows examples of non-trivial statistical structures, for which $\hat{\nabla} A=0$. The most famous is that on the hypersurface of $\mathbf{R}^{n+1}$ given by equation $x_{1} \cdot \ldots \cdot x_{n+1}=1$ for $x_{1}>0, \ldots, x_{n+1}>0$. This hypersurface is locally strongly convex affine sphere with $\hat{\nabla} A=0$, see [6]. By Theorem 4.1 neither the induced connection $\nabla$ nor its dual are complete. On the other hand, the Blaschke metric on this hypersurface is complete, see [6]. The class of Blaschke hypersurfaces satisfying the condition $\hat{\nabla} A=0$ turns out to be especially interesting. In the paper [4], all such hypersurfaces were classified. By Theorem 4.1, we know that in all non-trivial cases the induced connection is not complete.

Note that the compact case of hypersurfaces satisfying the condition $\hat{\nabla} A=0$ is not interesting. Namely, for an ovaloid this condition implies that the ovaloid must be an ellipsoid with its trivial affine structure. More precisely, we have

Proposition 4.3 Let: $M \rightarrow \mathbf{R}^{n+1}$ be an equiaffine ovaloid with the induced statistical structure $(g, \nabla)$. If $\hat{\nabla} A=0$, then the structure is trivial and the ovaloid is an ellipsoid.

Proof Since $\hat{\nabla} A$ is symmetric, the structure is conjugate symmetric, and consequently, the hypersurface is an equiaffine sphere and the Ricci tensor Ric is symmetric. It follows that $d \tau=0$, where $\tau(X)=\operatorname{tr} K_{X}$. Hence there is a function $\rho$ on $M$ such that $\tau=d \rho$. There is a point $p \in M$ at which $\rho$ attains an extremum. Then $\tau_{p}=0$. Since $\hat{\nabla} K=0$, we have $\hat{\nabla} \tau=0$ and consequently $\tau=0$ on $M$. It means that the hypersurface is a compact Blaschke affine sphere. It is well known from the classical affine differential geometry that such a sphere must be an ellipsoid with its trivial structure.

We shall now prove a positive result, that is, a theorem showing how to produce complete affine connections. 
Theorem 4.4 Let $(M, g)$ be a complete Riemannian manifold and A a cubic form given by

$$
A(X, Y, Z)=\alpha_{1} g(X, Y) d \sigma(Z)+\alpha_{2} g(Y, Z) d \sigma(X)+\alpha_{3} g(X, Z) d \sigma(Y)
$$

for some function $\sigma$ on $M$ and real numbers $\alpha_{1}, \alpha_{2}, \alpha_{3}$ such that $\alpha=\alpha_{1}+\alpha_{2}+\alpha_{3} \geq 0$. Assume that the function $\sigma$ is bounded from below on $M$. Let $K$ be a $(1,1)$-tensor field defined by formula $g(K(X, Y), Z)=A(X, Y, Z)$. Then, the connection $\nabla=\hat{\nabla}+K$ is complete.

Proof Let $\gamma:[0, b) \rightarrow M$ be a $\nabla$-geodesic such that $l(0)=1$. We have $A(\dot{\gamma}, \dot{\gamma}, \dot{\gamma})=\alpha d \sigma(\dot{\gamma}) g(\dot{\gamma}, \dot{\gamma})=\alpha(\sigma \circ \gamma)^{\prime} l^{2}$. Set $L=l^{2}$. We proved in (11) that $L^{\prime}=-2 A(\dot{\gamma}, \dot{\gamma}, \dot{\gamma})$. Hence, $(\ln L)^{\prime}=-2 \alpha(\sigma \circ \gamma)^{\prime}$, that is, $L(t)=e^{-2 \alpha \sigma(\gamma(t))+2 \alpha \sigma(\gamma(0))}$. If $\sigma \geq N$ on $M$, then $L(t) \leq e^{2 \sigma(\gamma(0))} e^{-2 \alpha N}$. We can now apply Propositions 3.5 and 3.2.

In particular, we have Noguchi's theorem from [9].

Corollary 4.5 Let $(M, g)$ be a complete Riemannian manifold and A a cubic form given by $A=\operatorname{sym}(d \sigma \otimes g)$ for some function $\sigma$ on $M$. Assume that the function $\sigma$ is bounded from below on $M$. Then, the statistical connection of the statistical structure $(g, A)$ is complete.

Note that if the function $\sigma$ is bounded from above, then $-\sigma$ is bounded from below and, consequently, the dual connection $\bar{\nabla}$ is complete. In particular, we have

Corollary 4.6 Let $(M, g)$ be a compact Riemannian manifold. Each function $\sigma$ on $M$ gives rise to a statistical structure whose statistical connection and its dual are complete. The cubic form of the structure is given by $A=\operatorname{sym}(d \sigma \otimes g)$.

\section{Completeness of the induced connections on equiaffine hypersurfaces}

It is known that pre-geodesics of induced connections on Blaschke affine spheres are obtained by intersecting the spheres with affine planes incident with the center of the sphere, see [8]. A more general statement holds. Namely, we have

Proposition 5.1 Let $f: M \rightarrow \mathbf{R}^{n+1}$ be a hypersurface equipped with a centroaffine normalization or with a constant transversal vector field. The image by $f$ of each geodesic of the induced connection is contained in a certain affine plane containing the center of the centroaffine normalization or, respectively, is parallel to the constant transversal vector field. Conversely, if the image by $f$ of a curve in $M$ lies in some affine plane containing the center of the centroaffine normalization or, respectively, is parallel to the constant transversal vector field, then the curve is a pre-geodesic of the induced connection.

Proof Denote by $\xi$ the transversal vector field. Let $\gamma(t)$ be a geodesic relative to the induced connection $\nabla$. By the Gauss formula (3) we have $D_{\dot{\gamma}} f_{*} \dot{\gamma}=g(\dot{\gamma}, \dot{\gamma}) \xi_{\gamma}$. The Weingarten formula says that $D_{\dot{\gamma}} \xi=\lambda f_{*} \dot{\gamma}$ for some real number $\lambda$. Denote by $\pi_{t}$ the affine plane passing 
through $f(\gamma(t))$ and whose direction is spanned by the vectors $f_{*} \dot{\gamma}(t)$ and $\xi_{\gamma(t)}$. The plane field $\pi_{t}$ is parallel relative to the connection $D$ and locally contains $f \circ \gamma$, hence it is constant along $\gamma$. The entire curve $f \circ \gamma$ lies in the plane.

Conversely, assume that for a curve $\gamma(t)$ its image by $f$ lies in a certain affine plane containing the center of the centroaffine normalization or, respectively, being parallel to the constant transversal vector field. Of course, $(f \circ \gamma)^{\prime}(t)$ and $(f \circ \gamma)^{\prime \prime}(t)$ must lie in the direction of the plane for every $t$. Such a plane also contains all transversal vectors $\xi_{\gamma(t)}$. The direction of the plane is spanned by $(f \circ \gamma)^{\prime}(t)$ and $\xi_{\gamma(t)}$ for every $t$. Therefore, by the Gauss formula, we have $\nabla_{\dot{\gamma}} \dot{\gamma}=\varphi \dot{\gamma}$ for some function $\varphi$ along $\gamma$. Thus $\gamma(t)$ is a $\nabla$-pre-geodesic.

Note that a hypersurface from the above theorem does not have to be non-degenerate. The following lemma will needed.

Lemma 5.2 Let $r(t)$ for $t \in \mathbf{R}$ be a curve in the vector space $\mathbf{R}^{2}$ such that the function $\operatorname{det}(r(t), \dot{r}(t)) \neq 0$ is bounded from 0 by a nonzero number on the whole of $\mathbf{R}$. There is a reparametrization of the curve being a diffeomorphism of $\mathbf{R}$ and such that after the reparametrization $\ddot{r}$ is parallel to r everywhere on $\mathbf{R}$.

Proof By replacing the parameter $t$ by $-t$, if necessary, we can assume that $\operatorname{det}(r(t), \dot{r}(t))>0$ and this function is bounded from 0 by some positive number for all $t \in \mathbf{R}$. We can write

$$
\ddot{r}(t)=\alpha(t) \dot{r}(t)+\beta(t) r(t) .
$$

Set $\rho(t):=\ln \operatorname{det}(r(t), \dot{r}(t))$. One sees that

$$
\rho^{\prime}=\frac{\operatorname{det}(r, \ddot{r})}{\operatorname{det}(r, \dot{r})}=\alpha .
$$

We are looking for a reparametrization $s(t)$ such that $(r(t(s)))^{\prime \prime}$ is parallel to $r(t(s))$ for every $s$. We have

$$
\begin{aligned}
& (r(t(s)))^{\prime}=\frac{d t}{d s} \dot{r}(t(s)) \\
& (r(t(s)))^{\prime \prime}=\left(\frac{d t}{d s}\right)^{2} \ddot{r}(t(s))+\frac{d^{2} t}{d s^{2}} \dot{r}(t(s)) \\
& \quad=\left(\frac{d t}{d s}\right)^{2}[\alpha(t(s)) \dot{r}(t(s))+\beta(t(s)) r(t(s))]+\frac{d^{2} t}{d s^{2}} \dot{r}(t(s)) .
\end{aligned}
$$

We want that

$$
\alpha(t(s))\left(\frac{d t}{d s}\right)^{2}+\frac{d^{2} t}{d s^{2}}=0
$$

The last equality can be written as

$$
\left(\rho(t(s))^{\prime} \frac{d t}{d s}+\frac{d^{2} t}{d s^{2}}=0 .\right.
$$

Denote the function $t(s)$ by $\varphi$ for a moment. Assume that $\varphi^{\prime}>0$ everywhere. We have $(\rho \circ \varphi)^{\prime}=-\frac{\varphi^{\prime \prime}}{\varphi^{\prime}}$ and hence $\rho \circ \varphi=-\left(\ln \varphi^{\prime}\right)+C$, where $C$ is a constant. Hence $\varphi^{\prime}=e^{-\rho \circ \varphi} e^{C}$ and consequently 


$$
\frac{d s}{d t}=e^{\rho} e^{-C}=\operatorname{det}(r, \dot{r}) e^{-C}
$$

In order to get a desired reparametrization, it is now sufficient to integrate the function $\operatorname{det}(r, \dot{r}) e^{-C}$. The anti-derivative is strictly increasing. Since $\operatorname{det}(r, \dot{r})$ is bounded from zero by some positive number, using (32) we get $\frac{d s}{d t}>N$ for some positive number $N$. It follows that $s(t) \rightarrow \infty$ for $t \rightarrow \infty$ and $s(t) \rightarrow-\infty$ if $t \rightarrow-\infty$, that is, the reparametrization is a global diffeomorphism of $\mathbf{R}$.

When we have a hypersurface of $\mathbf{R}^{n+1}$ with a centroaffine normalization, then we can assume that the center of the normalization is $0 \in \mathbf{R}^{n+1}$. An affine plane passing through 0 is a vector plane and we can assume that it is endowed with some arbitrary chosen determinant det. We can also assume that the transversal vector field is equal to the minus position vector field relative to the center. We have

Theorem 5.3 If for a given hypersurface $f: M \rightarrow \mathbf{R}^{n+1}$ endowed with a centroaffine normalization with center at $0 \in \mathbf{R}^{n+1}$ each curve for lying in a plane $\pi$ containing 0 can be parametrized globally on $\mathbf{R}$ as $\gamma(t)$ in such a way that the function $\operatorname{det}\left((f \circ \gamma)(t),(f \circ \gamma)^{\prime}(t)\right)$ is bounded from zero by a nonzero number for all $t \in \mathbf{R}$, then the induced connection $\nabla$ on $M$ is complete.

Proof By Proposition 5.1 we know that for every $\nabla$-pre-geodesic its image by $f$ lies in a certain plane $\pi \ni 0$. We can parametrize this curve in $\pi$ as in Lemma 5.2. Assume that $f \circ \gamma$ is such a global parametrization. We can write $D_{\dot{\gamma}} f_{*} \dot{\gamma}=(f \circ \gamma)^{\prime \prime}=\rho(f \circ \gamma)$ for some function $\rho$ along $\gamma$. On the other hand $D_{\dot{\gamma}} f_{*} \dot{\gamma}=f_{*} \nabla_{\dot{\gamma}} \dot{\gamma}-g(\dot{\gamma}, \dot{\gamma})(f \circ \gamma)$. It follows that $\nabla_{\dot{\gamma}} \dot{\gamma}=0$.

We can now prove

Theorem 5.4 On a centroaffine ovaloid in $\mathbf{R}^{n+1}$, the induced connection and its dual are complete.

Proof An ovaloid is a non-degenerate hypersurface modeled on a Euclidean sphere, but it is automatically a strongly convex imbedding of a Euclidean sphere. Hence, we can assume that it is, in particular, a subset of $\mathbf{R}^{n+1}$. A pre-geodesic of the induced connection is a curve diffeomorphic to the ordinary circle lying in some plane $\pi$. Take a periodic regular parametrization $\gamma(t)$ of a pre-geodesic defined on the whole of $\mathbf{R}$. Then, the function $\operatorname{det}(\gamma, \dot{\gamma})$ is nowhere zero. Since it is periodic, it is bounded from 0 by a nonzero number. We can now use Theorem 5.3. Since the affine shape operator for a centroaffine ovaloid is a nonzero multiple of the identity, the conormal hypersurface is also locally strongly convex. Hence, it is an ovaloid with the standard centroaffine normalization. The dual connection is the induced connection for the centroaffine conormal ovaloid.

Instead of an ovaloid, one can consider a convex (not necessarily strongly) imbedding of a Euclidean sphere. When we have its centroaffine normalization, then we see, as in the above theorem, that the induced connection is complete.

When we have an ovaloid in $\mathbf{R}^{n+1}$ equipped with any equiaffine transversal vector field, then the conormal map is an immersion but not necessarily non-degenerate, that is, the conormal, in general, is not an ovaloid in the dual space. But if the shape operator $S$ for the 
given ovaloid is non-singular everywhere, then the conormal is a non-degenerate immersion and therefore it is a centroaffine ovaloid. Hence, by Theorem 5.4, we obtain

Theorem 5.5 For an ovaloid in $\mathbf{R}^{n+1}$ equipped with any equiaffine transversal vector field, whose affine Gauss curvature $\operatorname{det} S$ is nowhere zero, the dual connection is complete.

Lemma 5.3 can be also applied to non-compact manifolds as well.

Example 5.6 Consider an elliptic paraboloid in $\mathbf{R}^{3}$. After choosing a suitable coordinate system $(x, y, z)$, the paraboloid can be viewed as the graph of the function $z=x^{2}+y^{2}-1$. Consider the centroaffine normalization with center at $0 \in \mathbf{R}^{n+1}$. If we intersect the paraboloid by a plane passing through the center, we obtain either an ellipse or a parabola. The ellipses can be treated as in the proof of Theorem 5.4. Considering the parabolas, it is sufficient to look at the parabola $z=x^{2}-1$ on the plane $y=0$. Its easiest parametrization is $\gamma(t)=\left(t, 0, t^{2}-1\right)$ for $t \in R$. We have $\operatorname{det}(\gamma(t), \dot{\gamma}(t))=t^{2}+1$. We can now apply Theorem 5.3.

Using Theorems 5.4 and 2.3 one gets

Theorem 5.7 Let $(g, \nabla)$ be a statistical structure on a manifold $M$ diffeomorphic to a Euclidean sphere.

1) If the Ricci tensor of $\nabla$ is symmetric non-degenerate and $\nabla$ is projectively flat, then $\nabla$ is complete on $M$.

2) If the structure is conjugate symmetric and the connection $\nabla$ is projectively flat, then $\nabla$ and its dual connection $\bar{\nabla}$ are complete on $M$.

Proof 1) Since Ric is symmetric, so is $\overline{\mathrm{Ric}}$. The dual connection for $\bar{\nabla}$ is equal to $\nabla$ and this connection is projectively flat. We can now apply Theorem 2.3 for $(g, \bar{\nabla})$. We get an ovaloid $f: M \rightarrow \mathbf{R}^{n+1}$ with some equiaffine transversal vector field, the induced connection $\bar{\nabla}$ and the second fundamental form $g$. Take the conormal map $\bar{f}$ equipped with the standard centroaffine normalization. Then, $\nabla$ is the induced connection for this centroaffine conormal immersion. Let $h$ be the second fundamental form for the centroaffine conormal immersion. The shape operator for the centroaffine conormal immersion is id. The Gauss equation (for the centroaffine conormal) is the following

$$
R(X, Y) Z=h(Y, Z) X-h(X, Z) Y .
$$

The projective flatness of $\nabla$ implies

$$
R(X, Y) Z=\frac{1}{n-1}(\operatorname{Ric}(Y, Z) X-\operatorname{Ric}(X, Z) Y)
$$

for $n>2$. For $n=2$, the above equality always holds. Hence, $h=\frac{1}{n-1}$ Ric is non-degenerate, and therefore, the centroaffine conormal immersion is a centroaffine ovaloid with the induced connection $\nabla$. We can now apply Theorem 5.4. 
2) For a conjugate symmetric statistical structures both statistical connections $\nabla$, $\bar{\nabla}$ are Ricci-symmetric. By Proposition 2.2 both connections are projectively flat. By Theorem 2.3 the statistical structure $(g, \nabla)$ can be realized on an ovaloid. Since the statistical structure is conjugate symmetric, the ovaloid is an equiaffine sphere. It means that it is a centroaffine ovaloid and, by Theorem 5.4, $\nabla$ and $\bar{\nabla}$ are complete.

As concerns 1 ) in the above theorem note that, by Theorem 5.4, the connection dual to $\nabla$ relative to $h=\frac{1}{n-1}$ Ric is also complete, but it is not the connection $\bar{\nabla}$ in general. In the context of Theorem 5.7, let us recall the structure on the torus from Example 3.14. That structure is conjugate symmetric, the statistical connection is projectively flat, its Ricci tensor is non-degenerate, and the connection is not complete. The dual connection is not complete either.

Open Access This article is licensed under a Creative Commons Attribution 4.0 International License, which permits use, sharing, adaptation, distribution and reproduction in any medium or format, as long as you give appropriate credit to the original author(s) and the source, provide a link to the Creative Commons licence, and indicate if changes were made. The images or other third party material in this article are included in the article's Creative Commons licence, unless indicated otherwise in a credit line to the material. If material is not included in the article's Creative Commons licence and your intended use is not permitted by statutory regulation or exceeds the permitted use, you will need to obtain permission directly from the copyright holder. To view a copy of this licence, visit http://creativecommons.org/licenses/by/4.0/.

\section{References}

1. Dillen, F., Nomizu, K., Vrancken, L.: Conjugate connections and Radon's theorem in affine differential geometry. Monat. Math. 109, 221-235 (1990)

2. Calabi, E.: Complete affine hypersurfaces I. Symposia Math. 10, 19-38 (1972)

3. Cheng, S.Y., Yau, S.T.: Complete affine hypersurfaces, Part I, The completeness of affine metrics. Commun. Pure Appl. Math. 39, 839-866 (1986)

4. Hu, Z., Li, H., Vrancken, L.: Locally strongly convex affine hypersurfaces with parallel cubic form. J. Differ. Geom. 87, 239-307 (2011)

5. Kobayashi, S., Nomizu, K.: Foundations of Differential Geometry, vol. I. John Wiley and Sons, New York (1963)

6. Li, A.-M., Simon, U., Zhao, G.: Global Affine Differential Geometry of Hypersurfaces. W. de Greuter, Berlin-New York (1993)

7. Nomizu, K.: On completeness in affine differential geometry. Geom. Dedic. 20, $43-49$ (1986)

8. Nomizu, K., Sasaki, T.: Affine Differential Geometry. Cambridge University Press, Geometry of Affine Immersions (1994)

9. Noguchi, M.: Geometry of statistical manifolds. Diff. Geom. Appl. 2, 197-222 (1992)

10. Opozda, B.: Bochner's technique for statistical structures. Ann. Glob. Anal. Geom. 48, 357-395 (2015)

11. Schneider, R.: Zur affinen Differentialgeometrie im Grossen I. Math. Z. 101, 375-406 (1967)

12. Trudinger, N., Wang, X.-J.: Affine complete locally convex hypersurfaces. Invent. Math. 150, 45-60 (2002)

Publisher's Note Springer Nature remains neutral with regard to jurisdictional claims in published maps and institutional affiliations. 